\title{
Contação de Histórias em Unidade Oncológica Pediátrica
}

doi: https://doi.org/10.32635/2176-9745.RBC.2018v64n3.44

Storytelling in Pediatric Oncology Unit
La narración de Historias en Unidad de Oncología Pediátrica

Sabrina de Jesus Oliveira Neves'; Patrícia Fernandes do Prado²

Resumo

Introduçáo: Em oncologia pediátrica, inúmeros e complexos desafios estão presentes, muitos relacionados às mudanças existenciais que permeiam o mundo da criança e da família e daqueles que profissionalmente cuidam desses pacientes. Objetivo: Identificar os benefícios da contação de histórias no cuidado à criança oncológica. Método: Trata-se de um estudo de revisão integrativa da literatura, no qual os artigos foram pesquisados, no período de 2003 a 2018, nas línguas portuguesa e inglesa, nas bases de dados: Literatura Latino-Americana e do Caribe em Ciências da Saúde (Lilacs), Bases de Dados de Enfermagem (Bdenf), Medical Literature Analysis and Retrieval System Online (Medline), Scientific Electronic Library Online (SciELO) e Centro Nacional de Informaçáo de Ciências Médicas de Cuba (Cumed). Resultados: Dos artigos encontrados, três estavam de acordo com a proposta da temática. Entre os benefícios da contação de histórias na pediatria oncológica, destacam-se o bem-estar dos pacientes, a colaboração no processo saúde-doença aliviando a dor e o sofrimento proveniente do tratamento oncológico, além do favorecimento do vínculo entre a criança com a equipe multiprofissional. Conclusáo: $\mathrm{A}$ contação de história é uma estratégia que possibilita à criança com câncer um maior conforto em relaçáo ao seu estado clínico, maximiza o seu bem-estar e ajuda em sua recuperação, permitindo um cuidado mais humanizado.

Palavras-chave: Criança; Neoplasias; Biblioterapia; Ludoterapia.

\begin{abstract}
Introduction: In pediatric oncology, innumerable and complex challenges are present, many related to the existential changes that permeate the world of the child and the family and those who professionally care for these patients. Objective: Identify the benefits of storytelling in oncological child care. Method: This is an integrative review of the literature, in which the articles were searched from 2003 to 2018, in the Portuguese and English languages, in the databases: Latin American and Caribbean Literature in Health Sciences (Lilacs), Nursing Databases (Bdenf), Medical Literature Analysis and Retrieval System Online (Medline), Scientific Electronic Library Online (SciELO) and National Information Center of Medical Sciences of Cuba (Cumed). Results: Of the articles found tree were in accordance with the proposal of the theme. Among the benefits of storytelling in oncology pediatrics are patient well-being, collaboration in the health-disease process, relieving pain and suffering from oncological treatment, and favoring the bond between the child and the team multi-professional. Conclusion: The historytelling is a strategy that enables children with cancer to be more comfortable with their clinical condition, maximize their well-being and help in their recovery, allowing for more humanized care. Key words: Child; Neoplasms; Bibliotherapy; Play Therapy.
\end{abstract}

Resumen

Introducción: En oncología pediátrica, innumerables y complejos desafíos están presentes, muchos relacionados a los cambios existenciales que permean el mundo del niño y de la familia y de aquellos que profesionalmente cuidan a esos pacientes. Objetivo: Identificar los beneficios de la cuenta de historias en el cuidado al niño oncológico. Método: Se trata de un estudio de revisión integradora de la literatura, donde los artículos se buscaron 2003-2018, los idiomas portugués e inglés, en las bases de datos: América Latina y el Caribe Ciencias de la Salud (Lilacs) Bases de Datos de Enfermería (Bdenf), Sistema de análisis y recuperación de literatura médica en línea (Medline), Biblioteca electrónica científica en línea (SciELO) y el Centro Nacional de Información de Ciencias Médicas de Cuba (Cumed). Resultados: De los artículos encontrados tres estaban de acuerdo con la propuesta de la temática. Entre los beneficios de la cuenta de historias en la pediatría oncológica, se destaca el bienestar de los pacientes, la colaboración en el proceso saludenfermedad aliviando el dolor y el sufrimiento proveniente del tratamiento oncológico, además del favorecimiento del vínculo entre el nińo con el equipo múltiples. Conclusión: La cuenta de historia es una estrategia que permite al niño con cáncer un mayor confort en relación a su estado clínico, maximiza su bienestar y ayuda en su recuperación, permitiendo un cuidado más humanizado.

Palabras clave: Niño; Neoplasias; Biblioterapia; Ludoterapia.

\footnotetext{
${ }^{1}$ Universidade Estadual de Montes Claros. Montes Claros (MG), Brasil. Orcid iD: https://orcid.org/0000-0003-3311-9705 ${ }^{2}$ Universidade Estadual de Montes Claros. Montes Claros (MG), Brasil. Orcid iD: https://orcid.org/0000-0002-5433-5485 Endereço para correspondência: Sabrina de Jesus Oliveira Neves. Rua Manoel Rodrigues Lima, 260 - Nossa Senhora Aparecida. Bocaiuva (MG), Brasil. CEP 39390-000. E-mail: sabrinajesusoliveira@hotmail.com.
} 


\section{INTRODUÇÃO}

Em oncologia pediátrica, inúmeros e complexos desafios estáo presentes, muitos relacionados às mudanças existenciais que permeiam o mundo da criança e da família e daqueles que profissionalmente cuidam desses pacientes. O estigma, os aspectos negativos relacionados aos sintomas e o sofrimento atribuído ao câncer ampliam o desconforto a esse diagnóstico, que é uma das realidades mais difíceis a ser enfrentada por uma criança e seus familiares ${ }^{1}$.

No contexto hospitalar, durante o período de internação para o processo de tratamento do câncer, a criança sofre por estar longe de casa e de tudo que é comum em suas rotinas diárias; sobretudo, são submetidas a procedimentos dolorosos e invasivos, como a quimioterapia e seus efeitos colaterais, que podem afetar seu desenvolvimento físico, intelectual e emocional visto como uma barreira na luta contra enfermidades da rotina hospitalar ${ }^{2}$.

Partindo dessas alteraçóes, ocorridas repentinamente na vida da criança, nota-se a importância de projetos essenciais para a superaçáo dos aspectos negativos que o câncer traz, incluindo uma assistência adequada que vise, por meio de açóes lúdicas, a minimizar os efeitos da hospitalização e a prevenir o estado emocional do paciente, a fim de promover o melhor relacionamento com o familiar e o profissional frente à internação ${ }^{3}$.

A contação de histórias caracteriza-se como uma dessas atividades lúdicas que não oferecem limitação para a criança, sendo prazerosa e também terapêutica. Contar uma história é mais que diversão e estímulo da imaginação, proporciona o desenvolvimento do raciocínio, auxiliando na compreensáo de situaçóes desagradáveis e ajudando a resolver conflitos ${ }^{4}$.

Essa atividade contribui para que o ambiente hospitalar, considerado como "lugar de doença", se torne um ambiente alegre, favorecendo a aprendizagem e estimulando a leitura. Além disso, o acesso às histórias infantis e aos livros propicia uma boa interaçáo com os contadores de história, que passam a ser importantes no processo de enfrentamento da doença e na busca pelo restabelecimento da saúde 5 .

Assim, na oncologia pediátrica, o lúdico, expressado por meio de histórias, é um instrumento terapêutico que auxilia o cuidado a criança, facilitando sua adaptaçáo e aceitação ao tratamento e, ainda, amenizando sentimentos como dor, medo, irritabilidade e angústia ${ }^{6}$.

Considerando tais pressupostos, cabe aos profissionais da saúde aprofundarem-se nessa temática, que tem ganhado espaço na literatura nos últimos anos, compreendendo a importância do lúdico dentro das unidades hospitalares e a utilização dessa terapêutica como uma forma positiva e criativa que abarque as incapacidades da criança para lidar com as dificuldades postas pelo câncer. Desse modo, delimita-se o seguinte questionamento de pesquisa: Qual o benefício da atividade de contar histórias no cuidado à criança com câncer?

Este estudo tem como objetivo identificar os benefícios da contação de histórias no cuidado à criança oncológica.

\section{MÉTODO}

O estudo caracteriza-se como uma revisão integrativa, que é um método que proporciona reunir e sintetizar resultados de pesquisas sobre um delimitado tema ou questão, com o objetivo de aprofundamento e integração do conhecimento e possível aplicabilidade dos estudos na prática ${ }^{7,8}$.

As estratégias de busca sobre a questão da pesquisa foram definidas em um protocolo que norteou a construção do trabalho. As fases da revisão integrativa foram: definição do tema e da questáo norteadora; critérios para a seleçâo dos estudos; definiçâo das informaçôes e categorização dos estudos; avaliação dos estudos; e, finalmente, a interpretação dos resultados e apresentação da revisão ${ }^{8}$.

O levantamento de artigos relacionados a essa temática foi realizado nos periódicos indexados nas bases de dados: Literatura Latino-Americana e do Caribe em Ciências da Saúde (Lilacs), Bases de Dados de Enfermagem (BDENF), realizado por meio do portal da Biblioteca Nacional de Saúde (BVS), Scientific Electronic Library Online (SciELO), Medical Literature Analysis and Retrieval System Online (Medline) e Centro Nacional de Informação de Ciências Médicas de Cuba (Cumed), nos meses de julho a agosto de 2018, utilizando os descritores: criança, oncologia, leitura e lúdico. Os descritores foram cruzados e combinados usando os operadores booleanos "e" e/ou "and".

Os critérios de inclusão para a seleção do estudo foram: artigos completos disponíveis eletronicamente, cuja população-alvo era pediátrica ( 0 a 19 anos) com câncer; divulgados em língua portuguesa, inglesa e espanhola, em periódicos nacionais e internacionais entre os anos de 2003 e 2018. Os critérios de exclusão foram: artigos em duplicidade, tese, e os que não respondiam à problemática desta pesquisa.

Foram encontrados 257 estudos relacionados ao tema, sendo que a seleçâo dos artigos foi realizada inicialmente a partir da procura das palavras-chave no título ou no resumo e, quando necessário, consultou-se o texto. Foram excluídos 254 estudos e a amostra da presente revisão foi constituída por três publicaçôes. As estratégias de busca utilizadas nas respectivas bases de dados e os motivos da exclusão foram apresentados no fluxograma (Figura 1), como recomendado pelo grupo Prisma? . 


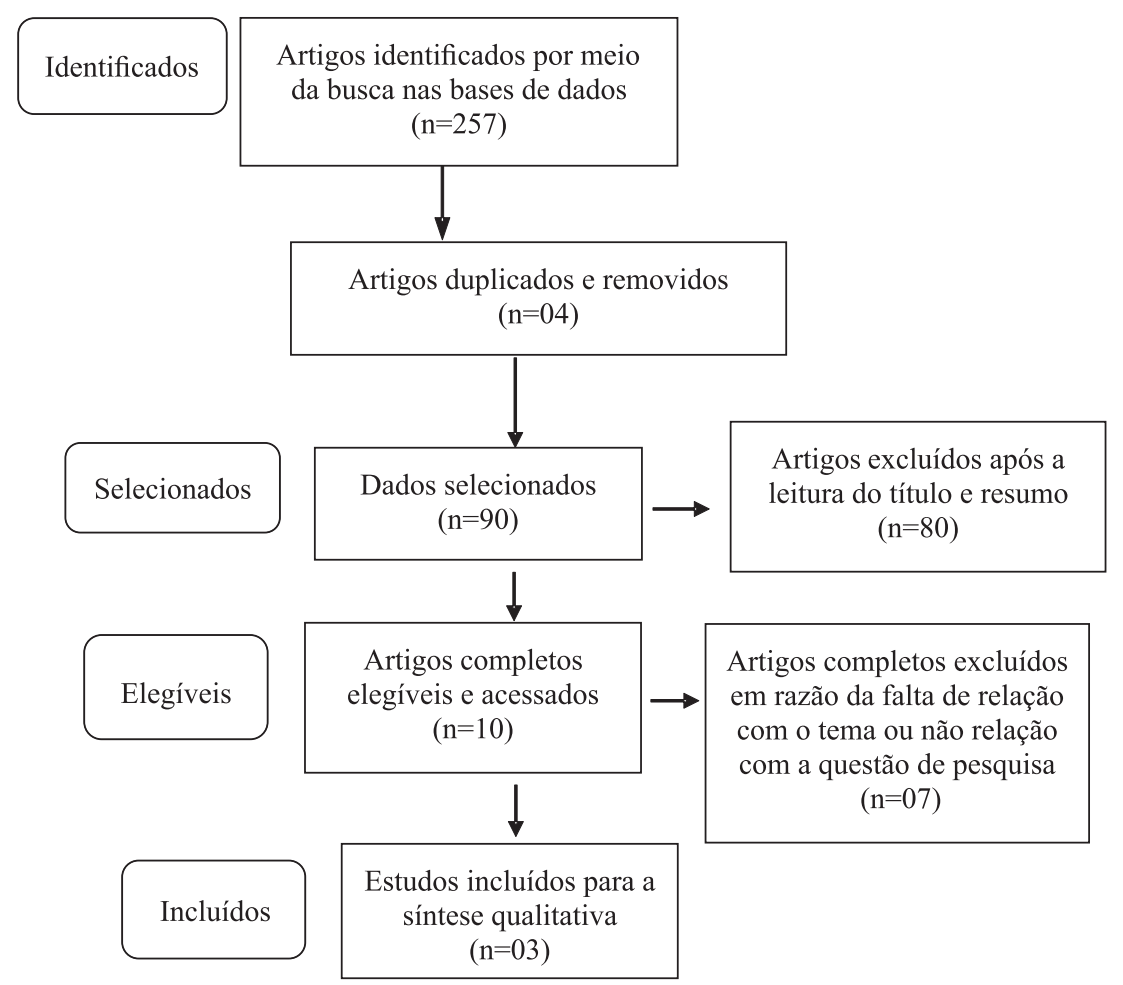

Figura 1. Fluxograma, segundo Prisma, para seleção dos estudos encontrados, Montes Claros, MG, Brasil, 2018 Fonte: Adaptado, Grupo Prisma?.

Este estudo não apresentou a necessidade de aprovação pelo Comitê de Ética em Pesquisa, uma vez que manipula dados de livre acesso, não se tratando, portanto, de documentos que requeiram sigilo ético.

\section{RESULTADOS}

O Quadro 1 apresenta as características dos artigos selecionados e utilizados na revisão integrativa como nome do autor, título do trabalho, periódico, população/ participantes; metodologia, tamanho da amostra e principais resultados do estudo.

\section{DISCUSSÃO}

Contar história na pediatria desperta encantos e sensaçóes de alegria aos pacientes, bem como aceitaçáo daquele marco traumático, e ainda contribui para seu desenvolvimento cognitivo, afetivo e social ${ }^{10}$.

O estudo de Paixão, Damasceno e Silva revelou que utilizar abordagem lúdica como a contação de histórias para aqueles pacientes pediátricos que são restritos ao leito e/ou apresentam locomoçáo prejudicada decorrente da enfermidade colabora no processo saúde-doença e alivia a dor e o sofrimento proveniente do tratamento oncológico $^{11}$.
Para tanto, contar história é uma estratégia lúdica que permeia os corredores das pediatrias oncológicas buscando o bem-estar dos pacientes, uma vez que ouvir resgata sonhos e esperanças, além de acelerar o processo de recuperaçáo do paciente, diminuir o tempo de internação, criar vínculo com a equipe multiprofissional e vivenciar o cuidado humanizado ${ }^{10}$.

As histórias permitem viver muito além da nossa realidade, dando a oportunidade de refletir sobre os nossos próprios problemas, solidificando, assim, a ideia de que crianças hospitalizadas precisam se abstrair do momento em que vivem. Entre os contos de fadas, Pedrosa et al. destacam os livros: A Bela e a Fera, que propicia a integração entre o fácil e o difícil, uma situação bela (a infância) e outra feroz (o tratamento), porém tendo um final feliz. Os Três Porquinhos, que representa a passagem pelas fases da vida com um final feliz (casa de palha infância; casa de madeira - adolescência; e casa de tijolo - fase adulta), apesar dos problemas (lobo) ${ }^{12}$.

Constatou-se que há poucos estudos na literatura nos últimos anos que abordam a prática de contar histórias como estratégia utilizada em oncologia pediátrica. Portanto, é notório que novas investigaçôes devam surgir, com o intuito de coletar mais informaçóes sobre o tema abordado, para assim qualificar a assistência prestada a esse público. 
Quadro 1. Distribuição dos artigos segundo autores, título, periódico, população/participantes; metodologia, tamanho da amostra e principais resultados, 2003- 2018

\begin{tabular}{|c|c|c|c|c|c|c|}
\hline Autor & Tí́tulo & Periódico & $\begin{array}{l}\text { População/ } \\
\text { participantes }\end{array}$ & Metodologia & $\begin{array}{l}\text { Tamanho da } \\
\text { amostra }\end{array}$ & Principais resultados do estudo \\
\hline Silva et al. ${ }^{10}$ & $\begin{array}{l}\text { Rodas de sonho } \\
\text { e imaginação: } \\
\text { contando histórias } \\
\text { em um serviço de } \\
\text { quimioterapia } \\
\text { infantil }\end{array}$ & $\begin{array}{l}\text { Rev Enferm } \\
\text { UFSM }\end{array}$ & $\begin{array}{l}\text { Enfermeiras, } \\
\text { acadêmicos } \\
\text { de medicina, } \\
\text { enfermagem e } \\
\text { psicologia }\end{array}$ & $\begin{array}{l}\text { Relato de } \\
\text { experiência }\end{array}$ & 21 integrantes & $\begin{array}{l}\text { Por meio da contação, é possível compreender } \\
\text { aspectos relacionados ao processo de } \\
\text { adoecimento, vivenciados pelo binômio } \\
\text { crianças-familiares e, desse modo, transformar } \\
\text { a prática do cuidado e tratamento. As } \\
\text { práticas lúdicas são principais ferramentas de } \\
\text { aproximação entre os profissionais de saúde e } 0 \\
\text { universo das crianças }\end{array}$ \\
\hline $\begin{array}{l}\text { Paixão, } \\
\text { Damasceno e } \\
\text { Silva }{ }^{11}\end{array}$ & $\begin{array}{l}\text { Importância das } \\
\text { atividades lúdicas na } \\
\text { terapia oncológica } \\
\text { infantil }\end{array}$ & $\begin{array}{l}\text { CuidArte } \\
\text { Enferm. }\end{array}$ & $\begin{array}{l}\text { Crianças nas fases } \\
\text { pré-escolar e } \\
\text { escolar }\end{array}$ & $\begin{array}{l}\text { Revisão } \\
\text { bibliográfica }\end{array}$ & $\begin{array}{l}\text { Não } \\
\text { especificado }\end{array}$ & $\begin{array}{l}\text { A leitura, entre outras estratégias lúdicas, como } \\
\text { brincadeiras, músicas, sons, teatralização, } \\
\text { danças, pinturas e desenhos, auxilia no } \\
\text { desenvolvimento, aprendizagem, socialização } \\
\text { e humanização, trazendo inúmeros benefícios } \\
\text { durante o processo de tratamento, recuperação } \\
\text { e reabilitação para a criança hospitalizada, } \\
\text { família e equipe multiprofissional }\end{array}$ \\
\hline Pedrosa et al. ${ }^{12}$ & $\begin{array}{l}\text { Diversão em } \\
\text { movimento: um } \\
\text { projeto lúdico } \\
\text { para crianças } \\
\text { hospitalizadas no } \\
\text { Serviço de Oncologia } \\
\text { Pediátrica do } \\
\text { Instituto Materno } \\
\text { Infantil Prof. } \\
\text { Fernando Figueira }\end{array}$ & $\begin{array}{l}\text { Rev. Bras. } \\
\text { Saúde Mater. } \\
\text { Infant. }\end{array}$ & $\begin{array}{l}\text { Crianças } \\
\text { internadas na } \\
\text { enfermaria } \\
\text { oncológica } \\
\text { pediátrica do IMIP } \\
\text { e acompanhantes }\end{array}$ & $\begin{array}{l}\text { Relato de } \\
\text { experiência }\end{array}$ & 60 pacientes & $\begin{array}{l}\text { Entre as possíveis estratégias utilizadas } \\
\text { pela criança para enfrentar o processo da } \\
\text { hospitalização, encontram-se o brincar e a } \\
\text { leitura, sendo essas práticas próprias de seu } \\
\text { momento de vida, nas quais ela descobre, } \\
\text { experimenta, inventa, exercita e confere suas } \\
\text { habilidades, além de terem estimuladas a } \\
\text { criatividade, a iniciativa e a autoconfiança. } \\
0 \text { desenvolvimento dessas estratégias torna o } \\
\text { ambiente hospitalar mais agradável à criança e } \\
\text { permite, de forma favorável, a continuidade do } \\
\text { desenvolvimento humano }\end{array}$ \\
\hline
\end{tabular}

\section{CONCLUSÃO}

A contação de história é uma estratégia que possibilita à criança com câncer um maior conforto em relaçáo ao seu estado clínico, maximiza o seu bem-estar e pode ter efeitos terapêuticos para a criança durante o período de tratamento oncológico, fazendo com que a mesma possa recriar seu estado emocional, favorecendo o estabelecimento de vínculos com os profissionais do cuidado.

Entretanto, tem sido um método ainda pouco utilizado pela equipe de saúde, sendo escassos os estudos dedicados à compreensão da estratégia de contar história como fator fundamental ao desenvolvimento humano. Portanto, os profissionais que cuidam dessa clientela devem reconhecer essa necessidade, propiciando meios para a sua realização e incorporando-os na assistência, permitindo um cuidado mais humanizado.

\section{CONTRIBUIÇÕES}

Sabrina de Jesus Oliveira Neves contribuiu na concepção e planejamento do estudo, obtenção, análise e interpretação dos dados, redaçáo do manuscrito e aprovação final da versão publicada. Patrícia Fernandes do Prado contribuiu na concepção e planejamento do estudo, análise e interpretação dos dados, redação do manuscrito, revisão crítica e aprovação final da versão publicada.

\section{DECLARAÇÃO DE CONFLITO DE INTERESSES}

Nada a declarar.

\section{FONTES DE FINANCIAMENTO}

Não há. 


\section{REFERÊNCIAS}

1. Teixeira RP, Ramalho WS, Fernandes ICF, Salge AKM, Barbosa MA, Siqueira KM. A família da criança com câncer: percepçôes de profissionais de enfermagem atuantes em oncologia pediátrica. Cienc Cuid Saude. 2012 Out-Dez;11(4):784-791.

2. Silva LCA, Gracindo RF, Calheiros MIF, Oliveira VF, Souza JPG. O efeito terapêutico do ato de ouvir e contar histórias em um setor de tratamento oncológico: relato de experiência. Gep News. 2017 Out-Dez;1(4):71-76.

3. Nicolino TNA, Barbieri MC, Tacla MTGM, Ferrari RAP. Contação de história na unidade pediátrica: percepção de acompanhantes de crianças hospitalizadas. Rev Enferm UFSM. 2015 Jan-Mar;5(1):32-39.

4. Torres SM, Tettamanzy ALL. Contação de histórias: resgaste da memória e estímulo à imaginaçáo. Nau Literária. 2008 Jan-Jun;4(1):1-8.

5. Sousa MP, Nascimento AR, Araújo HML. Projeto era uma vez: promovendo a educação e humanizando o atendimento de crianças hospitalizadas com a contação de histórias. [Internet]. [acesso 2018 Jul 25]. Disponível em: http://www.editorarealize.com.br/revistas/fiped/ trabalhos/Comunicacao_19.pdf.

6. Depianti JRB, Silva LF, Carvalho AS, Monteiro ACM. Nursing perceptions of the benefits of ludicity on care practices for children with cancer: a descriptive study. Online Braz J Nurs. 2014;13(2):158-165.

7. Souza MT, Silva MD, Carvalho R. Revisão integrativa: o que é e como fazer. Einstein. 2010;8(1):102-106.

8. Mendes KDS, Silveira RCCP, Galvão CM. Revisão integrativa: método de pesquisa para a incorporação de evidências na saúde e na enfermagem. Texto \& Contexto Enferm. 2008 Out-Dez;17(4):758-64.

9. Moher D, Liberati A, Tetzlaff J, Altman DG, PRISMA Group. Preferred reporting items for systematic reviews and meta-analyses: the PRISMA Statement. PLoS Med. 2009 Jul;6(7):e1000097.

10. Silva SO, Morais GSN, Dias JA, Silva SPC, Pereira MS, Batista MSS. Rodas de sonho e imaginaçáo: contando histórias em um serviço de quimioterapia infantil. Rev Enferm UFSM. 2016 Jul-Set;6(3):434-441.

11. Paixão $A B$, Damasceno TAS, Silva JC. Importância das atividades lúdicas na terapia oncológica infantil. CuidArte Enferm. 2016 Jul-Dez;10(2):209-216.

12. Pedrosa AM, Monteiro H, Lins K, Pedrosa F, Melo C. Diversão em movimento: um projeto lúdico para crianças hospitalizadas no Serviço de Oncologia Pediátrica do Instituto Materno Infantil Prof. Fernando
Figueira, IMIP. Rev Bras Saúde Mater. Infant. 2007 Jan-Mar;7(1):99-106. 